\title{
¿Formación en la debilidad? (Selbst)-Aufhebung y Tú en Novalis, lector de Fichte*
}

\author{
Education towards Weakness? Selbstaufhebung and You in Novalis' \\ Study of Fichte
}

\author{
Giovanni Panno \\ giovanni.panno@gmail.com \\ (Universität Tübingen, Tübingen, Alemanha)
}

\begin{abstract}
Resumo: Hablar de la educación del Yo, es decir, de su formación, es hablar de la co-formación y co-educación del mundo. Traicionando y respetando al mismo tiempo el espíritu de la primera Wissenschaftslehre, Novalis muestra la correspondencia micromacrocósmica de Yo y Mundo. Para lograr esta correspondencia es necesaria una acción del Yo que, aparentemente, va en contra de su propia actividad, revelándose como una especie de "debilidad". El siguiente trabajo aplica la categoría de debilidad para interpretar el movimiento de Selbstentäußerung que hace posible la nueva posición necesaria de Yo que permitiría al mundo de ser simple mundo y al anthropos de ser macroanthropos. Este mismo mecanismo de debilidad es el que actúa en el contexto político (Glaube und Liebe) e histórico (Die Christenheit oder Europa).
\end{abstract}

Palabras clave: Novalis; Fichte; Tú; Keywords: Novalis; Fichte-Studien; You; formación; imaginación productiva.

\begin{abstract}
Novalis develops the Fichtean ideas of dialectic between I and Not-I showing a micro-macrocosmic relation between I and World. Education of I corresponds here to forming of I within the World. This paper applies the category of weakness to describe the new necessary position of I as a movement outside itself (Selbstentäußerung): this would allow the world to be world as such without being only a product of I. The paper shows further that the same mechanism of weakness is at work in a political (Glaube und Liebe) and historical context (Die Christenheit oder Europa).
\end{abstract}

DOI: http://dx.doi.org/10.11606/issn.2318-9800.v25i2p137-153

\footnotetext{
* Retomo y reelaboro aquí algunos temas de los cuales ya me había ocupado in G. Panno (2007), Relazione e Schweben nelle Fichte Studien di Novalis. Padova: Imprimitur, al que me permito remitir. Las citas de Novalis corresponden a la edición de Robert Caner-Liese (2007), Estudios sobre Fichte y otros escritos. Madrid: Akal (EF seguido de número de fragmento y página); para el Allgemeines Brouillon, dado que no existe traducción castellana, me baso en la edición italiana de Moretti y Desideri (Scritti Filosofici 2019, citada como SF, número de fragmento y página). En cualquier caso, siempre señalo la referencia a la edición crítica de Samuel y Mähl (NS, volumen, línea y página). Por la traducción agradezco el trabajo de Jorge Retamal, la preciosa ayuda de Carla Miotto. El texto se ha enriquecido con los comentarios de los presentes al Congreso Fichte y Educación, organizado por Oscar Cubo a quien expreso aquí mis agradecimientos.
} 
Nun, fragt ihr, und was zeigte sich ihm hier? Ich weiß es nicht [...]

Was er all da gesehen und erfahren, Hat seine Zunge nie bekannt. [...] „Weh dem, der zu der Wahrheit geht durch Schuld, Sie wird ihm nimmermehr erfreulich sein".

(Entonces, preguntáis, ¿qué se le mostró ahí? No los é $[\ldots .$.

Todo lo que él vio y experimentó allí Su lengua nunca lo dio a conocer. [...] "Ay de aquel que va a la verdad mediante la culpa, Ella nunca le será gratificante”).

Schiller, F. Das verschleierte Bild zu Sais. Philosophische Gedichte, 74.

\section{Introducción}

¿Me pongo en la medida que me sustraigo [aufhebe]? (EF 278, 112; NS II, 196, 4)

La propuesta novalisiana "statt $N[i c h t] I[c h]=D u$ " (SF 820, 876; NS III, 429$30,13)$ es el resultado de una parábola teórica concebida a partir de la lectura de la Grundlage der gesamten Wissenschaftslehre 1794/5 - de los llamados Fichte Studien (1795/6) - que aparece también en escritos de diversa naturaleza, incluidos algunos fragmentos posteriores (Blüthenstaub, 1797-8), el Allgemeines Brouillon (1798-9) y los escritos sobre el estado y la historia (Glaube und Liebe, 1797-8, y Die Christenheit, 1799).

El No Yo fichteano no solo es renombrado, sino elevado a Tú. Esto es posible porque Novalis elabora una nueva concepción del Yo, a cuya posición pertenece una autosustracción. No se trata aquí de una abolición del Yo: este continúa siendo «fundamento de la permanencia en aquello que es mutable - es principio de la multiplicidad suprema» y hace posibles las determinaciones, aun no siendo «límite y determinación» (SF II, 876, 820; NS III, 429-30, 13). Con la expresión 'formación en la debilidad' me refiero aquí al movimiento de sustracción que hace posible la relación "Yo-No Yo" como "Yo-Tú". Novalis utiliza expresiones diferentes para este proceso," pero la más precisa sería Selbst-Aufhebung, que es posible extrapolar de la siguiente observación:

Yo no soy en la medida en que me pongo, sino en la medida en que me supero [sondern inwiefern ich mich aufhebe] - Yo no soy en la medida en que estoy en mí, que me aplico a mí mismo (EF 278, 112, NS II, 196, 4).

1 Novalis utiliza los términos Selbstfremdmachung y Selbstveränderung (SF 820, 876; NS III 429, 13); Entäußerung (EF 566, 173; NS II, 270, 28); Selbstentäußerung (EF 26, 204, traducida como autoalienación NS II 24, 423); Alienation (SF 118, 396; NS II, 34, 551). 
En realidad, en esta alienación el Yo se forma, levantándose y enriqueciéndose al mismo tiempo. Con el término "Yo" Novalis entiende casi de inmediato anthropos, que será, entonces, en el estadio más amplio de su Bildung, un macroanthropos. Traicionando y respetando al mismo tiempo el espíritu de la primera Wissenschaftslehre y previendo proféticamente algunos resultados posteriores, como los del segundo 'Vortrag' de la Wissenschaftslehre 1804, ${ }^{2}$ Novalis muestra la correspondencia micromacrocósmica de Yo y Mundo. Hablar de la formación del Yo es, entonces, hablar de la co-formación del mundo.

Sin embargo, no es este el lugar para un análisis filológico específico de las apariciones fichtianas en los escritos de Novalis. Un trabajo de este tipo ha sido ya realizado con más o menos éxito por algunos intérpretes (Wanning, 1997; Loheide, 2000; Stanchina, 2002; Waibel, 2006; Panno, 2007). Tampoco es este el momento para discutir el sentido del "Yo - No Yo" en la primera Wissenschaftlehre; no solo por razones de espacio, sino por las razones temáticas ya mencionadas anteriormente. Por desgracia, no existen trabajos de referencia que tematicen el concepto de educación "en la debilidad" en relación con el primer romanticismo, por lo que me limitaré a señalar algunos textos esenciales para la comprensión de la perspectiva política del pensamiento novalisiano, ya que este es un ámbito claramente afín al aquí analizado (Schmitt, 1981, Panno, 2005). Central para la elaboración del presente tema han sido los textos de Desideri (1998), sobre el concepto de actividad-pasividad en la conciencia, y el de Frank (2002) sobre la conexión autoconocimiento-sentimiento de sí (entendido justamente en modo activo y pasivo).

Sin embargo, creo pertinente mencionar aquí algunas observaciones semánticas: se podría vincular el concepto de formación en la debilidad con el de Selbstbeschränkung del Fundamento del derecho natural (1796). La inspiración teórica de la división interna del Yo se modera en este contexto para convertirse en una autolimitación que garantiza una relación política. Con el término Selbstbeschränkung Fichte se refiere aquí a la sustracción del propiamente teórico ius in omnia y a la producción de un espacio que permita la libertad del Yo, partiendo de la hipótesis de que el otro hará lo mismo (Grundlage des Naturrechts, § 4). Ahora bien, en Novalis no se encuentra un uso similar de este concepto: la autolimitación como asunción de un ser libre por fuera del Yo es superada -no resuelta- por un concepto de Yo del que no se sale sino expandiendo paradójicamente su esfera al infinito.

Otro elemento que pudo haber sido determinante desde la primera lectura de Fichte, a saber, el Tú, aparece en Novalis en unas pocas líneas y, como veremos más adelante, únicamente en el Allgemeines Brouillon. Parece que Novalis se refiere, en particular, a un pasaje de la Segunda Introducción:

2 Me refiero aquí a las metáforas visisbles en el segundo „Vortrag“ de 1804 (véase V. Vortrag, SW 130, Meiner 53 y IX Vortrag, SW 156, Meiner 86). Novalis profundiza ya desde los Fichte Studien en el concepto de duplicación de la imagen y de la visión (EF160, 476; NS II 476, 255). 
El concepto del tú surge por medio de la unión del ello y del yo. El concepto del yo en esta oposición, o sea, como concepto del individuo, es la síntesis del yo consigo mismo. Lo que en el acto descrito pone a sí mismo, y lo que pone no en general, sino lo que pone como un yo, soy yo, y lo puesto en el mismo acto por mí, y no por sí mismo, como un yo, eres tú. De este producto de una síntesis a exponer puede abstraerse, sin duda alguna, pues lo que uno mismo ha sintetizado ha de poder también analizarlo. $Y$ lo que queda después de esta abstracción es el yo en general, es decir, lo no-objeto (Fichte, Segunda Introducción, p. 84).

Es posible que Novalis, en la lectura del Allgemeines Brouillon, postergase el debate con los críticos las ideas que Fichte desarrolla en este pasaje. Ciertamente uno de los puntos de tensión de la reflexión de Novalis es precisamente la producción de un no-objeto y la posibilidad de hacer emerger las cosas sin volverlas objeto -y esto no solo a nivel teórico, sino también antropológico en el sentido más amplio-. Pero este resultado, que aparece en los escritos posteriores a los Fichte Studien, es posible gracias al trabajo realizado allí en torno al concepto de negación - o de formación a la debilidad.

De acuerdo con lo anterior, el primer ámbito en el que es posible centrar la atención es la relación del Yo con sus propias subdivisiones internas y con el No Yo, es decir, la formación desde un nivel más estrictamente teórico. En segundo lugar, me ocuparé de la figura de una aparente alteridad, el tú respecto del "Yo".

Los dos ámbitos restantes que se pueden pensar esquemáticamente tratan del concepto de debilidad en la formación del Estado (3) y, en última instancia, de la idea de formación desde el punto de vista del devenir histórico (4). Estos ámbitos son subdivisibles para Novalis solo desde el punto de vista analítico, porque todos ellos se compenetran el uno con el otro.

\section{La relación del Yo con sus propias subdivisiones internas y con el No Yo}

1.1 «Representamos [lo idéntico] mediante su no-ser, mediante un no-idéntico» (EF 1,33 ; NS II 104, 6)

La estructura de la conciencia que Novalis elabora en los Fichte Studien muestra cómo para el Yo mismo, separado desde el principio de su ser puro, y en el Yo, imagen reflejada del ser, estos ámbitos son siempre estructurales respecto a un único movimiento de mediación. Esta emerge tanto más fuerte cuanto más se supera el Yo, compenetrado en el mundo, para hacerlo emerger, cuanto más expone, por decirlo así, su no, produciendo reflexivamente el movimiento originario de la posición (EF 278, 112; NS II, 196, 4).

El ser puro, elemento originario, es para Novalis aquello Idéntico que nosotros abandonamos para representarlo. Habiendo requerido la exteriorización de la cosa en sí, no podemos conocerla más que de modo negativo, como aquello que queda 
después de la operación de abstracción. Tal es el carácter del Yo, cuya determinación es estructuralmente posterior respecto al mero ser (nur Seyn), puesto que este mismo no está sujeto a ninguna determinación.

En el proceso de redenominación que recorre los Fichte Studien hay que considerar, entonces, el Yo puro, el Yo absoluto, el ser puro, el mero ser y la acción originaria misma (Urhandlung, EF 22, 48; NS II, 13, 119). Lo Idéntico que cede el lugar a su representación no manifiesta la presencia de 'propiedades', sino las de su representación.

1.2 Salida del ser puro: reflexión y sentimiento. Reflexión como negación del sentimiento

Siguiendo la relación proporcional que emerge reiteradamente en los Fichte Studien entre ser y representar, se puede decir que la acción originaria no sería la del pensamiento, sino la del sentimiento. De hecho, por un lado están el ser y el sentir, por otro, el pensar y el representar (cfr. EF 379, 140; NS II 22, 232). Estas dos últimas constituyen acciones de la alienación de la cosa en sí, pero no se trata de una exteriorización que ocurra en un hipotético 'otro' del Yo. En efecto, el saber (en el cual incluyo aquí provisionalmente sin diferenciarlos tanto el pensar como el representar) es definido como ser fuera del ser (absoluto, idéntico), pero aun siempre en el ser (SF 2, 36; NS II 106, 5). ¿A qué corresponde, entonces, este segundo ser, en el que ocurre la acción de salida de lo Idéntico y de la representación? Asumiendo que es imposible salir del círculo del Yo y que el único lugar donde este saber puede formarse es precisamente la conciencia, Novalis distingue entre un Yo absoluto y un Yo relativo, entre un Yo puro y un Yo que tiene tesis/antítesis/síntesis, que presenta divisiones internas, que sabe y que es presentante (y representante). La sustancia de tal Yo está constituida ciertamente por la reflexión, por lo tanto, en cuanto actividad en la que se presenta el Yo mismo. Sin embargo, esta no es la Urhandlung en la que Novalis identifica paradójicamente también una pasividad, pues el Yo percibe sus límites y de algún modo los padece: la conciencia es originariamente la conciencia de un sentimiento, la conciencia de sí, un sentirse (Selbstgefühl, cfr. Frank 2002, 233). En la acción originaria reflexión y sentimiento se pertenecen, y así se produce una «interacción del yo consigo mismo de un modo aparentemente indirecto» (EF 48, 22; NS II 119, 6).

Pensamiento y representación pierden al Yo como sentimiento, exponiéndolo en cuanto sentimiento atravesado por la reflexión. Por lo tanto, esta representación es el no del Yo en su completitud de sentimiento activo y reflexión pasiva, es decir, aún no concretada. En la dinámica del intercambio, que más adelante analizaremos brevemente, a la actividad de la reflexión le corresponde la pasividad del sentimiento, 
en el sentido de que este, en cuanto reflexionado, se presenta según la categoría de saber, que arroja fuera del ser originario - pero siempre dentro del ser del Yo -. El sentimiento es, entonces, el No (Yo) de la reflexión, que a su vez es el No (Yo) del sentimiento. Al primer Yo absoluto, por lo tanto, constituido por sentimiento y reflexión, se opone aquí un Yo relativo; más bien se podría decir que hay dos, pero solo a nivel hermenéutico, porque, de hecho, al primero se le opone cada vez solo un Yo. La misma esfera-ser es la esfera conciencia cuando la reflexión se opone al sentimiento - la división interna del Yo que así se forma es denominada por Novalis Yo analítico -. En oposición al Yo analítico, el Yo sintético recoge, en un proceso reflexivo ulterior, tanto la primera reflexión en la que fueron producidos (separados) sentimiento y reflexión, como la $x$ de donde provienen separadamente como una abstracción del uno y del otro y como producto de la imaginación.

Separar y unir, tal como la diaíresis platónica del Sofista (Platon, Soph. 226d-e y $248 c-d$ ), son aquí verbos de una acción de oposición que saca a la luz el ser a través de su no. Por lo tanto, en la estructura de la conciencia hay relaciones recíprocas en las cuales un extremo determina al otro. Incluso el Yo sintético es, a su vez, solo producto de una operación de reflexión en la conciencia, no es absoluto, sino schlechthin sólo una idea. Naturalmente, este juego impone a Novalis una ampliación ulterior de los límites del Yo, de manera que para incluir el plexo constituido por el Yo analítico (sentimiento + reflexión distintos del ser puro) y el Yo sintético (Yo analítico + conciencia de la salida de la esfera de lo Idéntico), los fragmentos posteriores del Allgemeines Brouillon invoca a un gran Yo=Tú (SF II, 398, 771; NS III 314, 19).

Recorrer las divisiones internas del Yo corresponde a una exposición de la acción originaria en su irrepresentabilidad o, mejor dicho, en su ficcionalidad, mostrarlo en su plexo de «verdad, ficción o apariencia [Schein]» (EF 234, 97; NS II 179, 23).

Abandonamos lo idéntico para exponerlo [Wir verlassen das Identische um es darzustellen]. 0 esto sólo sucede de forma aparente -y la imaginación consigue que nos lo creamos- sucede lo que ya es [es geschieht, was schon ist] -naturalmente, gracias a un separar y unir imaginarios- o lo representamos mediante su no-ser, mediante un no-idéntico [Oder wir stellen es durch ein Nichtseyn, durch ein Nichtidentisches vor] -un signo- algo determinado para algo análogamente determinante (EF 1,33; NS II 104,6$).^{3}$

La conexión entre estos niveles debe ser construida: el Yo que conecta es obra de la imaginación (EF 553, 169; NS II 13, 265), de manera que la filosofía opera como producción de una imagen trascendental en cuanto arte de la composición (SF 847, 881; NS III 14, 433). El medio de tal arte es, ordo inversus, la reflexión de

\footnotetext{
3 El Hölderlin del fragmento sobre el «significado de las tragedias», datado en 1803, en el cual el signo debe ser $=0$ para que lo Originario se represente, es sin duda intérprete de esta necesidad, que por ello se configura como trágica. El Urpunkt tiene que volverse entonces un Nullpunkt husserliano, llevando en un movimiento de reflexión a aquellos límites de la conciencia que deben ser reconstruidos artísticamente. Lo Idéntico novalisiano, lo Originario hölderliniano, desde siempre perdidos, son, de hecho, un lugar solo sentido. Cfr. Hölderlin, SW, Band X, 93.
} 
la reflexión, que permite poner en evidencia cómo se juega la construcción de la conciencia a partir de una serie de contraposiciones y ayudar a la re-articulación.

\subsection{Wechsel y Schweben}

Hasta ahora el no ha aparecido como estructural a la historia de la conciencia en su desarrollo natural de salida del puro ser, del Yo absoluto fichteano, y, en este sentido, como sustracción estructural de lo Idéntico. Por otra parte, las acciones que la conciencia lleva a cabo para poder definirse, denominarse 'conciencia', no descansan en una apofática, sino en la exposición de este no como condición de la construcción misma. En tanto que ficticia, esta imagen de sí basada en el no del ser es la única cosa que la conciencia puede tener de sí. A la intuición y a la reflexión las acompaña necesariamente la producción de aquella imagen que es la conciencia, función de aquella Einbildungskraft (imaginación) ya definida por Fichte como un Schweben (oscilar) entre determinación y no determinación, entre finito e infinito (Fichte, Grundlage, 217).

La novedad de la lectura novalisiana radica en el vínculo entre el momento imaginativo y esa libertad que mueve al Yo en el intercambio. También en este caso el término medio es la negación o, mejor dicho, la negación determinada del Yo mismo. Partiendo del principio de que «ser intercambio y determinación de intercambio son una y la misma cosa» (EF 284, 118; NS II 32, 203), y que el Yo es en la medida en que se efectúa el intercambio, Novalis se enfrenta al problema de esta inalcanzable infinitud asumiendo la oscilación como punto de producción de la realidad misma.

Ser libre es la tendencia del yo - la capacidad de ser libre es la imaginación productiva - la armonía es la condición de su actividad - del oscilar entre opuestos [...]. De este punto luminoso de la oscilación fluye toda la realidad [...] La yoidad o la fuerza immaginativa productiva, el oscilar - determina y produce los extremos entre los cuales se oscila [...] (EF 555, 169; NS II 234-5, 555).

Si el Yo es capaz de ser este punto, no hay aquí una infinitud maligna, ni un regreso al infinito, ni tampoco un recurso al momento de lo sublime que es extrínseco al tiempo y al espacio. En este sentido, esta recuperación del pasaje fichteano de los Fichte Studien tiene un resultado, una repercución más allá de este conjunto de estudios: la oscilación entre opuestos produce toda la realidad, porque es en ella donde el Yo es libre, es decir, libre de expandirse en todas las direcciones, pero, sobre todo, libre de su ser mero Yo. Para ser la potencia de sí mismo debe, en efecto, sustraerse. La Aufhebung ya propuesta en los Fichte Studien se convierte en los fragmentos posteriores en la Selbsttödtung como acto filosófico por excelencia (EF 54, 291; NS II 1, 395). Solo de este modo el Yo puede entrar en una relación universal y ser intercambio (EF 455, 153; NS II 247, 19). «En el mundo temporal, el ser es 
una relación rítmica» (EF 456, 153; NS II 247, 28)», esto es, un modo de vibración/ oscilación entre los dos extremos, donde la posición está siempre vinculada a la contraposición.

En cuanto lugar de la oscilación, la yoidad incluye una reflexión determinada, libre -que va del No Yo al Yo- y otra también libre que va del Yo al No Yo. Los dos Yoes son, en un mismo movimiento, Inteligencia y Yo puro. Su actividad se separa de la esencia del Yo y permite una nueva compenetración. Pero ¿cuál de los diversos Yoes aquí presentados está llamado a aplicar sobre sí mismo el no de la Selbstaufhebung)?

\subsection{Libertad e imaginación productiva}

Explotando la fuerza formativa simbólica de la imaginación el Yo en cuanto tal puede elevarse más allá de su Allzumenschlickeit en el momento en que se hace él mismo símbolo («Fuerza formadora simbólica. Imaginación» EF 226, 92; NS II 5, 171). En este sentido, la imaginación es realmente el bien más grande y la fuerza que lleva a trascender la relación de objetivación unidireccional respecto al objeto-yo y al objeto-mundo, aun siendo, al mismo tiempo, inmanente (EF 325, 134; NS II 26, 224). Ambos ámbitos son internos y externos al Yo, por un lado, porque no hay salida del yo; por otro, porque ambos presentan límites sobre los cuales la reflexión se convierte en auto-reflexión y la conciencia puede volverse auto-conciencia. Conectar estos límites e intercambiar lo interno-externo significa revelar una interdependencia presente desde siempre, pero oculta bajo la presunta dirección de la actividad desde lo interno hacia lo externo (EF 630, 183; NS II, 11, 282).

El yo siempre es uno consigo mismo y siempre es lo opuesto de sí - es Uno y lo mismo dicho [...]. La autoconciencia sólo está aparentemente ligada a un individuo determinado [...]. La perfección más elevada de la inteligencia pensante consiste en ser libre incluso respecto de aquello que nos resulta extraño, lo dado, en asimilar libremente, esto es, en permitir que lo recibido llegue a ser algo autónomo y eficaz, algo libre, lo extraño como algo libre (EF 646, 188; NS II 286, 30).

Se puede comprender, entonces, el fichtismo sin golpe (ohne Anstoß) como aquella superación del límite interno, en términos fichteanos, gracias a la cual la fórmula Yo se desarrolla y se expande (SF 639, 832; NS III 385, 12). Se conserva ciertamente su contraposición ontológica, pero ya no se trata de contraponerse al No Yo «in seinem Sinn», sino de un movimiento compuesto en el que se reconoce el Schein de la imagen de sí producida por el Yo y del ser del que deriva, para negar la sustancialidad a este Yo, poniéndolo como abstracto en el juego de reflejo con su No Yo. Para la Entfremdung es necesaria la primera Entäußerung, allí donde el Yo no se reconoce como otro de un hipotético No Yo externo, sino de la imagen de sí. El Anstoß, en realidad, no es aquí tout court evitado, sino que es reconocido como 
un evento interno al Yo. Entonces, el Yo debe reconocer, hysteron proteron, que el primer momento por sí corresponde, en realidad, a un segundo momento, porque la reflexión que lo ha colocado como Yo ya ha tenido lugar antes de que se viese contrapuesto a un No. De este modo, el primer dato es la salida de sí, como se ha señalado más arriba, entendida como una negación que, a su vez, es reflejada (de algún modo negada) para obtener, via abstractionis, el original.

De este modo, ¿cómo puede ser libre el Yo? Novalis indica la necesidad de ser libre en lo ajeno. Pero incluso este fremd no es otra cosa que una falta de comunicación del Yo consigo mismo o, mejor dicho, una falta de actividad de la imaginación productiva que no ha tenido la fuerza de levantar el velo de la diosa de Sais. ${ }^{4}$

No se trata, en efecto, de una producción de alteridad, sino más bien de un desvelamiento de lo extraño presente en lo proprio. Ciertamente es difícil no tener presentes las categorías psicológicas freudianas de lo Unheimlich, ya que el mismo Novalis a menudo involucra en el discurso sobre el Yo el discurso de este yo sujeto (cfr. Freud, Das Unheimliche, 249). No obstante, el carácter del no es primariamente ontológico -esto podría ayudar a sortear el riesgo psicologizante no solo en los Fichte Studien, sino también en los escritos posteriores-, porque la conciencia niega estructuralmente su verdad originaria para poder representarse, tarea que le es propia, sustrayéndose a sí misma, para desarrollar así aquella esfera polar en la que sujeto y objeto se intercambian (SF 634, 831; NS III 10, 384). La auto-conciencia plenamente desarrollada no será, entonces, aquella que toma posesión del mundo expandiéndose y com-prendiéndolo, sino aquella que ve el mundo como un gran Yo, con el que se establece una relación con las propias divisiones. Por lo tanto, es esta experiencia de lo negativo lo que permite la exposición de este nexo microcosmosmacrocosmos, como intercambio entre lo interno y lo externo del mundo-Tú.

\section{Yo-Tú}

\subsection{Contra un Yo de buenos sentimientos}

El punto de fuga de la subjetividad de los Studien es a menudo identificado con una formación orientada a superar las relaciones internas del Yo en una suerte de inspiración intercultural (cfr. Buber 1993, Waldenfels 1997). Analizando las dos ocurrencias más significativas del término Tú en el Allgemeines Brouillon, el fragmento 398 y el fragmento 820, intentaré mostrar cómo la realización de una

\footnotetext{
4 Así lo muestra la narración gnóstica con la cual Novalis responde a Schiller, dejando que Hyazint, protagonista de una salida de lo propio y de un alejamiento de la amada para cruzar lo diverso, descubra detrás del velo de la diosa el rostro de la amada (o a sí mismo, según un pasaje de los paralipomena a los Discípulos). Con menos suerte (el mutismo o la muerte) terminaba el camino del viandante en Schiller. Cfr. Schiller, Philosophische Gedichte, p. 72-74 y Novalis, NS I, 108-9.
} 
relación recíproca (Wechselwirkung) no objetivante no está dirigida a una formación del Yo, sino a una formación del mundo a través del Yo. No obstante, este "a través de" debe hacerse como una mediación no mediada, sin poder suprimirse realmente en cuanto tal.

El Yo común [das gew(önlilche) Ich] y el Tú común son solo suplementos. Cada Tú es un suplemento del gran Yo. Nosotros no somos, de hecho, un Yo -pero podemos y debemos volvernos Yo-. Somos gérmenes del volverse-Yo [Wir sind Keime zum Ich werden]. Tenemos transformar todo en un Tú -en un segundo Yo-; solo de esa manera nos elevamos al gran Yo -que es Uno y Todo al mismo tiempo [Eins und Alles zugleich] (EF 398, 771; NS III 20, 314; trad. Carla Miotto).

Del segundo fragmento, el n. 820, se le suele dar importancia solo a la segunda parte, que indica el paso del No Yo al Tú («Du - Statt Nicht Ich - Du»), pero es precisamente la primera la que le confiere sentido a esta fórmula, que de lo contrario se reduciría a un mero Zauberwort:

Nosotros nos vemos en el sistema como miembros -es decir, en una línea ascendente y descendente, desde lo infinitamente pequeño hasta lo infinitamente grandehombres con infinitas variaciones. Nosotros comprendemos naturalmente todo aquello que es extraño solo mediante el autoextrañamiento [Selbstfremdmachung] la autotransformación - la autoobservación [...] (EF 820, 876; NS III 429-30, 13; trad. Carla Miotto).

No se supone aquí una fuga -efectivamente romántica- del Yo, sino una autotransformación que vuelve verdadera la Wechselwirkung de la Doctrina de la ciencia (Fichte, Grundlage, 268). Resulta necesario, entonces, un doble movimiento de descomposición del Yo empírico en su posición como absoluto y en su recomposición artística integrada del Tú. Ambos movimientos son propios de la filosofía novalisiana, que se configura como arte de la división del Yo empírico y su reunión con el gran Yo, a condición de un gesto hacia el mundo que debe trascender la actividad misma -y este es el verdadero elemento de proyección del pensamiento novalisiano sobre algunas poéticas del siglo XX, como las de Rilke y Pessoa-. Por lo tanto, constituye un error ver en la romantización del mundo una mera proyección del Yo; pero también sería inadecuado interpretar el pasaje del No Yo al Tú como una abdicación del Yo, una suerte de debilidad última de la posición. Ambos movimientos son necesarios: la expansión del Yo va de la mano con su autorreducción en tanto que subjetivación. El Yo se debe hacer referencia jeroglífica, porque en el símbolo es posible asumir su objetivización y su autoreducción.

\subsection{Lo verdadero es lo Entero - un entero múltiple (EF 234, 100; NS II, 177, 5)}

El movimiento de salida de sí y la compenetración del Mundo parece establecer una dialéctica triádica casi hegeliana. Sin embargo, lo que se podría reconocer como hegeliano ante litteram en Novalis, forzando los cánones de una correcta 
interpretación histórico-conceptual, es la verdad de lo entero, desde siempre presente, pero nunca completamente accesible, salvo en la Verwirklichichung de la esfera polar, en la cual los opuestos remiten el uno al otro.

La totalidad, en la que también la androginia se vuelve superación de la malvada infinitud que separa Yo y No Yo, es pensada a partir de una automodificación. Solo en el recorrido simultáneo de las dos vías, tanto hacia el interior como hacia el exterior, el Yo no absolutiza ninguna de ellas, manteniéndose en la oscilación que lo hace vital («La autoalienación es el origen de todo rebajamiento, como también, al contrario, el fundamento de toda verdadera elevación», EF 26, 204; NS II, 24, 423). Este andar en lo diverso, en lo desconocido, planteando una incógnita donde antes había un concepto sólido, es el movimiento de romantización que lleva al «Yo inferior» a ser identificado con el «mejor»y a reconocer una «serie cualitativa de potencias» (SF 105, 391; NS II, 545, 14) donde sólo parecía haber escisión. «Saltar encima/más allá de sí mismos» no es simple alienación, sino sustracción de sí respecto al movimiento que pone el mundo según este mismo yo.

A la Entäußerung como salida del Yo le corresponde una Entfaltung del Yo a través de la naturaleza como su cometido histórico. En una carta a A. W. Schlegel (NS IV, 245, 1), Novalis señala con el concepto de Entfaltung un elemento esencial del espíritu poético -allí donde lo poético, entendido como trascendental, está elevado a potencia y compenetrado filosóficamente-. El «misterio del bello despliegue» es una acción simultánea de intuición y descripción, de comprensión y creación, una suerte de Wechselwirkung (llamada en la carta Wechselvollendung), donde están unidas la acción hacia el interior (Hineinwirken) y la acción hacia el exterior (Herauswirken).

\subsection{Los Paralipomena en Sais: ¿un enfrentamiento imposible con la Sittenlehre?}

En el relato iniciático central de Los discípulos en Sais tiene lugar un juego de desvelamiento del Yo a través de una vía que se hace cargo del mundo. Hyacinth abandona lo originariamente suyo, a saber, a su amada Rosenblüthchen, para atravesar lo Fremd, de lo cual le había dejado huella la historia de un antiguo viajero. Al llegar al templo de la diosa de Sais, se atreve a levantar su velo, acto que ya en Schiller implicaba la afonía y la clausura con respecto a la relación con el mundo (cfr. Schiller, Philosophische Gedichte, pp. 65 y 71). Bajo el velo el protagonista de Novalis, no obstante, encuentra a su amada. En un pasaje de los Paralipomena a Los Discípulos en Saïs, Novalis ofrece una versión diferente del mismo final, según el cual Hyacinth se ve a sí mismo bajo el velo (NS I, 25, 66).

De la misma manera nos hablan otras importantes declaraciones poéticas, como las de Astralis, composición inserta en Heinrich von Ofterdingen (obra recientemente revalorizada por su fuerte carga panteísta y spinoziana). En la experiencia de quien 
sabe acoger en sí mismo lo diverso a través de la propia alienación, domina una idea de mezcla que no parece, sin embargo, dar razones de lo diverso especifico en el interior del producto. Ya no existe el reconocimiento de una alteridad que el Yo mismo produce, sino la conservación de la virtualidad en ella implicada como una suspensión sobre la ausencia de fundamento. ${ }^{5}$

Aquí se reconoce una de las dos principales dificultades presentes en la antropología novalisiana, junto a aquella vinculada a la estructura de la representación simbólica: el Schweben de la imaginación productiva parecería no conceder la posibilidad de una identidad permanente que no sea esa misma yoidad (Ichheit), en la que naturaleza y Yo, Tú y Dios se deben fundir. De hecho, parece ser prerrogativa exclusiva del genio poder mantener la unidad en la diversidad. El hombre de Novalis está llamado él mismo a hacerse genio, a «asimilar más individualidad» y transformarse «en un individuo sustancial», que requiere su artificialidad, su ser compuesto artísticamente.

Un hombre natural y modélico es el sueño de un poeta. Así pues, ¿qué nos queda? - la composición de uno artificial. Los hombres más excelentes de la nación se completan mutualmente. - En esta sociedad arde un espíritu social puro [...] (EF 66, 238; NS II, $502,10)$.

De hecho, al ser el genio la realización de la naturaleza intrínseca del Yo como Yo trascendental, su misma composición artística está más allá del artificio, constituye el retorno al hombre natural. El motivo plotiniano, que Novalis había elevado a numen tutelar próximo a Fichte, es evidente: se trata del retorno a ese Uno que conserva nuestra alma no descendida (Plotino, Enn. V, 6, 6). Aun así, el aspecto místico, no del todo ausente, es realizado en Novalis de otra manera: la tarea de romantizar el mundo no es una proyección hacia algo ultramundano, sino la liberación de la mundanidad (Weltlichkeit) de este mundo.

En este sentido, una comparación con Fichte, por ejemplo, con los Deberes del artista creador de obras de arte de el Sistema de la doctrina moral, pone a la vista más de un elemento en común: la formación del hombre en su unidad por medio del arte bello (Fichte, Sittenlehre, § 31). Del mismo modo, el cometido del filósofo para Fichte se corresponde, en parte, con la idea del genio novalisiano: «hacer común el punto de vista trascendental». Incluso el aspecto vagamente platónico del ideal, que el filósofo debe esforzarse por representar, no es extraño a Novalis, y no es del todo distinto el papel del arte en su aplicación educativo-política (cfr. Traub, 2006, p. 50), sobre todo por lo que respecta a la necesidad que el arte o, mejor dicho, que quien puede educar mediante el arte, contribuya a superar las formas esclerotizadas para liberar el espíritu.

\footnotetext{
5 Se establece, entonces, una dialéctica análoga a la baaderiana entre Begründung y Entgründung como elementos constitutivos de una reconciliación que en Novalis está presente y es infinita. Cfr. Baader, PhE p. 42.
} 


\section{Formar y educar al pueblo con Fe y amor [Glaube und Liebe]}

Cuando Novalis [habla del Estado como obra de arte] diciendo que el Estado es un macroanthropos, expresa un pensamiento milenario. El romanticismo radica precisamente en eso, en considerar dicho hombre-Estado como una «bella» individualidad, convirtiéndolo en objeto de amor y de sentimientos análogos (Schmitt, 1981, p.179, cfr. Novalis: SF 261, 747; NS III 286, 31).

El escrito novalisiano Glaube und Liebe, objeto de la crítica de Schmitt, no fue bien recibido por Federico Guillermo III; ciertamente no podía más que resultarle odiosa la idea de una monarquía republicana o de una república monárquica. No es que pueda sospecharse en Novalis un retorno al jacobinismo, pero incluso una interpretación literal del escrito bastó probablemente para aconsejar a Novalis que usara otro pseudónimo en caso de volver a publicar.

El tema de la superación de la forma estatal -o de una forma institucional respecto a su espíritu- no es nuevo, pero va acompañado de una utilización del símbolo (rey, pareja, monarquía) que es peculiar.

En este sentido Novalis retoma el tema de Algunas lecciones sobre el destino del sabio de Fichte: «el Estado, así como todo el resto de las instituciones humanas, que no son otra cosa que instrumentos, está dirigido hacia la propia autodestrucción: el propósito de todo gobierno radica en hacer superfluo al gobierno» (Fichte, Bestimmung des Gelehrten, p. 28). El mecanismo es el mismo que está en juego en la pareja iglesia invisible-iglesia visible del Kant de la Religión dentro de los límites de la mera razón y que Novalis tendrá especialmente presente en el texto sobre La Cristiandad (brevemente más adelante).

En Fe y amor el rey está llamado a ser más que rey, a saber, garante simbólico de la unidad del Estado más allá de su propio cargo. Es un rey que «se pone cada día la corona y su reconocimiento no precisa negociación» (EF 33, 232; NS II, 18, 494), y que por su valor representa un Yo trascendental, constituyendo así una referencia a lo que cada súbdito puede tomar como modelo. Aquí la forma adquirida, como la del rey, no debe constituir un obstáculo -se trata de una transposición de aquel Yo que debe suprimirse o, mejor dicho, debe superar simbólicamente su propia autorepresentación para comunicarse de verdad. Y para eso, primero debe ser reconocida como representación.

Todo el Estado conduce a la representación. Toda representación se basa en un hacer presente -lo no presente, y así sucesivamente - (fuerza milagrosa de la ficción). Mi fe y mi amor se basan en la fe representativa. Así, la hipótesis -la paz eterna ya está aquí- Dios está entre nosotros - América está aquí o en ningún sitio - la edad de oro está aquí - somos magos - somos morales, y ¿así sucesivamente? (SF 782, 868; NS III, 421, 16; trad. Carla Miotto). 
La reducción del todo a la representación del Yo no es un acto, sino un dato estructural, un límite que debe ser superado infinitamente como cometido moral. La «productividad esencialmente estética de los románticos» (Schmitt, 1981, 212) no constituye, por tanto, el diseño de una relación simbólica capaz de contener la alteridad, sino la representación de un metateatro político en el que el Yo intenta hacerse infinitamente otro: la estética es el instrumento asignado para esta tarea, en la que ella misma se funde con la moral y la política. Según Schmitt es precisamente la ausencia de una Trennung entre filosofía y política, la superposición de ambas, la que genera el paso de un ocasionalismo filosófico a una ineficacia política, al dejar caer en las manos de un tercero infinito la polaridad y la contradicción sin resolverla o enfrentarla de otra manera. «Para él [el romántico y ocasionalista] todo el mundo está suspendido dentro de Dios, su modo de pensar no es panteístico, sino panenteístico» (Schmitt, 1981, 141). De esta manera Schmitt comprende equivocadamente el concepto de símbolo en Novalis. Se trata de un tercero infinito, porque el sujeto que lo atraviesa no puede cristalizar el significado -la tarea de la formación, es decir, de la transformación del Yo y del mundo, a saber, del Mundo porque del Yo es necesariamente un cometido in-finito-. Esto conoce una representación simbólica en los periodos históricos abordados en Cristiandad o Europa.

\section{La superación de la letra en El Cristianismo (La Cristiandad o Europa)}

La narración introduce, primero en forma de fábula («es waren schönen glänzenden Zeiten», EF 243; NS III, 4, 507), el tema de una alternancia de épocas, definibles como positivas y negativas en relación con la misma Bildung, aunque no del individuo, ni de un pueblo en el interior del Estado, sino de la humanidad en cuanto tal. Estas diferentes épocas están marcadas por cesuras en las cuales el anquilosamiento de la época precedente se fractura por un movimiento revolucionario o de reforma. Los dos elementos que alternan aquí son letra y espíritu. Allí donde la letra sustituye al espíritu, tiene que haber una destrucción de la letra.

La verdadera anarquía es el elemento generador de la religión. De la destrucción de todo lo positivo alza su gloriosa cabeza como nueva fundadora universal [Weltstifterin] (EF 251; NS III, 517, 19).

El ritmo vital de la historia necesita una reforma con la condición de que ninguna de ellas se proponga como 'sistema' o como Iglesia, es decir, que permita la seducción del espíritu por la letra.

El medioevo de los tiempos auténticamente católicos (ächt katholischen Zeiten) es un momento fundamental de la historia, como lo sería, desde el ámbito teórico, la figura de lo Idéntico no diferenciado, lo inefable que se conoce a sí mismo sólo a costa de perderse como totalidad y de escindirse en partes. En cierto sentido, esto 
es lo que sucede con el texto sobre Europa: la totalidad subsumida jerárquicamente en un único jerarca remite a la ya citada primera observación de los Fichte Studien, según la cual «abandonamos lo idéntico para exponerlo [darstellen]» (EF 1, 33; NS II, 104, 6).

Si la separación en el interior del Cristianismo ha transformado la sede pontificia en trono, disputado luego por Estados surgidos de la desintegración de la unidad originaria, la Reforma, capaz de disolver lo cristalizado, se convierte ella misma en un elemento negativo desde el momento en que sustituye a la vieja letra por una nueva: el espíritu de la revolución, en cierto sentido, es sustituido por la estabilización de su movimiento. La introducción de la «filología» aplasta el espíritu en su posibilidad de movimiento, ya que busca iluminar aquello que, como «sentido de lo sagrado», debe mantenerse en penumbra, conservar su «misterio», no convertirse, como la traducción luterana lo permite, en un texto «popular». Lutero es culpable de establecer un paradójico «gobierno revolucionario permanente» (EF 247; NS III, 6, 512). Los efectos de la institucionalización de la Reforma son similares a aquellos que operan en la Ilustración, donde la filosofía es la que se contrapone a la fe:

El odio a la religión se extendió de forma natural y conseguente a todo aquello que fuera objeto de entusiasmo, se difamó el sentimento y la fantasía [...] y se convirtió la eterna música creadora del universo en el monótono tableteo de un enorme y mostruoso molino que, impulsado por la corriente de la causalidad, flota sobre la misma; un molino en sí, sin arquitecto ni molinero, en realidad un auténtico perpetuum mobile, un molino moliéndose a sí mismo (EF 250; NS III, 515, 23).

A este perpetuum mobile responde la romantización que debe reformar la cristalizada Ilustración. De hecho, solo en la aceptación de su debilidad es posible fundar una nueva filosofía.

Así como con la autosustracción del Yo, la formación en la debilidad de un complejo estatal y de una época les permite instaurar una relación verdadera y órganica con sus partes y el movimiento de la historia. Esto significa una liberación de las cristalizaciones y una comunicación macrocósmica y universal.

\section{Referencias}

\section{Fuentes primarias}

Baader, Filosofia Erotica: Baader F. Von. (1982). Filosofia erotica. Edición de L. Procesi Xella. Milano: Rusconi.

Fichte: Grundlage: Fichte, J.G. (1971). Grundlage der gesammten Wissenschaftslehre 1794. In: Fichtes Werke, I, ed. de I. H. Fichte. Berlin: de Gruyter.

Fichte: Segunda Introducción: Fichte, J.G. (1971). Zweite Einleitung. In: Fichtes 
Werke, I, ed. de I. H. Fichte. Berlin: de Gruyter. Trad. Primera y segunda introducción a la Teoría de la Ciencia. (1984). Ed. de J. Gaes. Madrid: Sarpe.

Fichte: Sittenlehre: Fichte, J. G. (1971). System der Sittenlehre. In: Fichtes Werke, ed. de I. H. Fichte. Berlin: De Gruyter (1834 ff.). Trad. it. Il sistema di etica secondo i principi della Dottrina della scienza. Ed. de C. de Pascale, Roma-Bari: Laterza 1994.

Fichte, J. G. (1979). Grundlage des Naturrechts nach Prinzipien der Wissenschaftslehre (1796). Hamburg: Meiner.

Fichte: Bestimmung des Gelehrten: Fichte, J. G. (1991). Einige Vorlesungen über die Bestimmung des Gelehrten. Trad. it. La missione del dotto. Pordenone: Studio Tesi (Editori Riuniti 1982).

Hölderlin: SW: Hölderlin, F. (1996). Sämtliche Werke. Historisch-kritische Ausgabe. Edición de D. E. Sattler. Frankfurt a. M.: Roter Stern 1974 ss. (Darmstadt: WBG 2004). Trad. it. de R. Ruschi, Scritti di estetica, Milano: Mondadori.

NS: Novalis (1960). Novalis Schriften. Edicción de R. Samuel, J. Mähl et a. Stuttgart: Kohlhammer 1960 ss.;

ES: Novalis (2007). Estudios sobre Fiche y otros escritos. Edición de R. Caner-Liese. Madrid: Akal.

SF: Novalis (2019). Opera filosofica. Edición de G. Moretti y F. Desideri. Torino: Einaudi 1993. Nueva edición Scritti filosofici. Brescia: Morcelliana.

Plotino: Enn.: Plotino (1999). Enneadi, edición de G. Faggin. Milano: Rusconi (1992).

Schiller: Philosophische Gedichte: Schiller, F.(1990). Philosophische Gedichte. Stuttgart: Reclam 1978, trad. it. de G. Moretti, Poesie filosofiche, Milano: SE.

\section{Literatura secundaria}

Brandt, A. (2010). "Weltbürgertum und Nationalidee in Fichtes Bildungskonzept". In: Stolzenberg, J. y Ulrichs, L. T. (ed. de). Bildung als Kunst: Fichte, Schiller, Humboldt, Nietzsche. Berlin: De Gruyter, pp. 59-68.

Buber, M. (1993). Il principio dialogico ed altri saggi. Milano: San Paolo.

De Mazza, M. E. (1999). Der verfaßte Körper. Zum Projekt einer organischen Gemeinschaft in der Politischen Romantik. Freiburg im Breisgau: Rombach.

Desideri, F. (1998). L'ascolto della coscienza. Una ricerca filosofica. Milano: Feltrinelli.

Frank, M. (2002). Selbstgefühl. Eine historisch-systematische Erkundung. Frankfurt a. M.: Suhrkamp.

Freud, S. (1992). Das Unheimliche. In: Gesammelte Werke. Edición de A. Mitscherlich, A. Richards, J. Strachey. Frankfurt am Main: Fischer 1982, Band XII, pp. 241-274. Trad. it. Il Perturbante. En: Opere 1905-1921. Roma: Newton \& Compton, pp. 1049-1070.

Loheide, B. (2000). Fichte und Novalis. Transzendentalphilosophisches Denken im romantisierenden Diskurs. Amsterdam-Atlanta: Rodopi.

Panno, G. (2005). "Urkönig-Urmensch: romanticismo politico e katechon del re". In: Ethics, pp. 55-81. 
Panno, G. (2007). Relazione e Schweben nelle Fichte Studien di Novalis. Padova: Imprimitur.

Schmitt, C. (1981). Politische Romantik. Berlin: Duncker \& Humblot 1919. Trad. it. Romanticismo politico, a cura di C. Galli. Milano: Giuffrè.

Summerer, S. (1974). Wirkliche Sittlichkeit und ästhetische Illusion. Die FichteRezeption in den Fragmenten und Aufzeichnungen F. Schlegels und Hardenbergs. Bonn: Bouvier V. Herbert Grundmann.

Stanchina, G. (2002). Il limite generante. Milano: Guerini e Associati.

Traub, H. (2006). Über die Pflichten des ästhetischen Künstlers. Der § 31 des Systems der Sittenlehre im Kontext von Fichtes Philosophie der Ästhetik. En: C. Asmuth y Wilhelm Metz (eds.). Die Sittenlehre J. G. Fichtes 1798-1812. Amsterdam, pp. 55-106.

Waibel, V. (2006). Filosofiren muß eine eigne Art von Denken seyn«. Zu Hardenbergs >Fichte-Studien< In: System und Systemkritik. Beiträge zu einem Grundproblem der klassischen deutschen Philosophie, hrsg. von Birgit Sandkaulen. Würzburg: Königshausen und Neumann, pp. 59-90.

Waldenfels, B. (1997). Topographie des Fremden. Frankfurt a. M.: Suhrkamp.

Wanning, B. (1997). Statt Ich-Du! Die Umwendung der Fichteschen Wissenschaftlehre ins Dialogische durch Novalis (Friedrich von Hardenberg). Fichte Studien, 12, 153-169.

Recebido em: 06.03.2020

Aceito em: 27.07.2020

Esta obra está licenciada com uma Licença Creative Commons Atribuição-NãoComercial-

-Compartilhalgual 4.0 Internacional.

https://creativecommons.org/licenses/by-nc-sa/4.0/

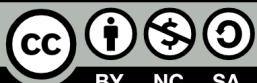

